\title{
VIVÊNCIAS DE CAMPO DO CURSO EPIDEMIOLOGIA APLICADA AOS SERVIÇOS DO SUS - EPISUS FUNDAMENTAL: RELATO DE EXPERIÊNCIA
}

\author{
Yuri Messias Lisboa \\ Universidade Federal do Recôncavo da Bahia - UFRB \\ yurimlisboa@gmail.com \\ Marta Souza Rocha \\ Secretaria de Saúde do Estado da Bahia - Sesab \\ mrocha37@hotmail.com \\ Thais Santana Mattos \\ Faculdade Santo Agostinho de Itabuna \\ thaisisameerah@gmail.com \\ Meire Núbia Santos de Santana \\ Universidade Estadual de Santa Cruz \\ meirenubia@yahoo.com.br
}

\begin{abstract}
Resumo
O presente trabalho teve por objetivo relatar a experiência de descrição das etapas do ciclo da vigilância no contexto das vivências de campo do Programa de Treinamento em Epidemiologia Aplicada aos serviços do SUS. Trata-se de um estudo descritivo, utilizou como instrumento de coleta um formulário para entrevista dos integrantes da equipe de saúde da família localizada no Distrito Sanitário da Boca do Rio, na cidade de Salvador - Bahia, no período de agosto a outubro de 2018. Foram identificados os pontos fortes e fracos intrínsecos da unidade de saúde da família, bem como as ameaças e oportunidades que dependem de outras instâncias administrativas da saúde ou de instituições de outros setores para o funcionamento do ciclo da vigilância. Também se estratificou as possíveis causas para o déficit/ausência de monitoramento das doenças diarreicas agudas, e por fim, classificou-se as circunstâncias de acordo com o grau de controle dos membros da equipe de saúde da família e realizou-se a devolutiva do trabalho para equipe de saúde afim de promover reflexão sobre as fases do ciclo de vigilância em saúde e apresentar recomendações de melhoria na qualidade das ações do mesmo. Conclui-se que apesar das unidades de saúde da rede básica serem os espaços privilegiados para o desenvolvimento das ações de vigilância, observa-se nesse trabalho de campo que a sistematização dos dados epidemiológicos que deveriam ser interpretados e atualizados rotineiramente pela equipe de saúde, ainda são ações realizadas por técnicos de outras instância, seja por profissionais da vigilância epidemiológica do Distrito Sanitário ou da Diretoria de Vigilância à Saúde da Secretaria Municipal de Saúde de Salvador. Destaca-se também a relevância da atividade de campo do EPI-SUS, enquanto atividade extensionista, para o desenvolvimento de uma reflexão crítica sobre a realidade por parte dos treinandos.
\end{abstract}

Palavras chave: Epidemiologia. Vigilância epidemiológica. Educação permanente. Serviços de saúde.

Cidadania em Ação: Revista de Extensão e Cultura, Florianópolis (SC), v.4, n.2, jul./dez.2020. 


\title{
FIELD EXPERIENCE IN THE COURSE OF EPIDEMIOLOGY APPLIED TO SUS SERVICES - EPISUS FUNDAMENTAL: EXPERIENCE REPORT
}

\begin{abstract}
The present article aimed to report the description experience's of the steps from the monitoring cicle in the perception field background of the Training Program in Applied Epidemiology for SUS services. It is a descriptive study, using a form for interview the participants of the health family team located in the Boca do Rio sanitary district, in Salvador - Bahia, during the time of August to October 2018. Were identified strenghts and intrisic points in the unit of health family, as well as threats and oportunities which depends on others health administrative instances or instituitons from differents sectors for the monitoring cicle performance. Also were stratified possible causes for the deficiency/absence of diarrhoeal acute diseases monitoring and finally, were classified the circunstances in accordance with the control grade of the team health family members and was held a feedback work for the team in order to promote a reflection about the monitoring cicle steps and show recommendations for improviment in the quality of it. It concludes that despite of the health units in primary care are a privileged space for development of monitoring actions, it is noticed in this work field that the systematization of epidemiological data that should be interpreted and updated routinely by the health team, are still actions performed by technicians from other instances, either by professionals from the epidemiological surveillance of the sanitary district or the Surveillance Directorate to Health Department of the Municipal Health Department of Salvador. The relevance of the EPI-SUS field activity, as an extension activity, is also highlighted for the development of a critical reflection on the reality by the trainees.
\end{abstract}

Keywords: Epidemiology. Epidemiological monitoring. Permanent Education. Health services.

\section{EXPERIENCIAS DE CAMPO DEL CURSO DE EPIDEMIOLOGÍA APLICADO A EL SUS - EPISUS FUNDAMENTAL: INFORME DE EXPERIENCIA}

\begin{abstract}
Resumen
Este estúdio tuvo como objetivo reportar la experiencia de describir las etapas del ciclo de vigilancia en el contexto de experiencias de campo del Programa de Capacitación en Epidemiología Aplicada a los servicios del SUS. Se trata de un estudio descriptivo, que utilizó como instrumento de recolección um formulário para entrevistar a membros del equipo de salud familiar ubicado en el Distrito Sanitario de Bocado Rio, en la ciudad de Salvador - Bahia, de agosto a octubre de 2018. Se identificaron las fortalezas y debilidades intrínsecas de la unidad de salud familiar, así como las amenazas y oportunidades que dependen de otras autoridades administrativas de salud o instituiciones de otros sectores para el funcionamento del ciclo de vigilancia. También se estratificaron las posibles causas del déficit/falta de seguimiento de las enfermedades diarreicas agudas, y finalmente se clasificaron las circunstancias según el grado de
\end{abstract}


control de los integrantes del equipo de salud familiar y se devolvió el trabajo para el equipo de salud con el fin de promover la reflexión sobre las fases del ciclo de vigilancia en salud y presentar recomendaciones para mejorar la calidad de sus acciones. Se concluye que si bien las unidades de salud de la red básica son los espacios privilegiados para el desarrollo de las acciones de vigilancia, se observa en este trabajo de campo que la sistematización de datos epidemiológicos que deben ser interpretados y actualizados de manera rutinaria por el equipo de salud, son acciones realizadas por técnicos de otras instancias, ya sea por profesionales de vigilancia epidemiológica del Distrito Sanitario o de la Dirección de Vigilanca em Salud de la Secretaría Municipal de Salud de Salvador. También se destaca la relevancia de la actividad de campo del EPI-SUS, como actividad de extensión, para el desarrollo de uma reflexión crítica sobre la realidad por parte de los participantes.

Palabras clave: Epidemiología. Monitoreo epidemiológico. Educación permanente. Servicios de salud. 


\section{INTRODUÇÃO}

O Programa de treinamento em Epidemiologia Aplicada aos Serviços do SUS (Epi-SUS) é uma estratégia de educação permanente que visa implantar no Brasil treinamentos em epidemiologia de campo, com enfoque na prática do serviço, e tem como objetivo aprimorar as habilidades dos profissionais de saúde fortalecendo a capacidade de resposta das unidades de saúde nas ações de controle das doenças e agravos à saúde da população, reconhecendo a importância de monitorar as condições de saúde através da vigilância em saúde (BRASIL, 2015).

Segundo Waldman (1998), a Vigilância em Saúde tem como objetivo a análise permanente da situação de saúde da população, e deve subsidiar o planejamento de ações e práticas que ajudem no enfrentamento dos problemas de saúde. Para sua operacionalização utiliza-se de instrumentos como o método epidemiológico (ROUQUAYROL, 1999) e um ciclo de funções específicas, com cinco componentes fundamentais - detecção, notificação, análise e interpretação, feedback e monitoramento - que permite conhecer e controlar os riscos, agravos e doenças da população de um determinado território (BRASIL, 2018).

Para que a vigilância aconteça é necessário existir casos suspeitos, definidos por critérios padronizados, e assim iniciar a investigação da ocorrência de determinada doença ou evento adverso à saúde. Estes são confirmados ou descartados conforme critérios adotados pelo do Ministério da Saúde. Com o objetivo de buscar fatores associados ao agravo, os casos devem ser categorizados de acordo com os dados de identificação, dados demográficos, clínicos, laboratoriais, contatos, dados do notificador (BRASIL, 2015).

Os casos suspeitos ou confirmados como doenças de notificação compulsória têm por base, a Lei 6.259 de 30 de outubro de 1975 e regulamentos que obrigam o médico e outros profissionais de saúde a notificar doenças de maneira mais ágil às autoridades sanitárias. A notificação compulsória consiste na comunicação da ocorrência de casos individuais ou surtos às autoridades sanitárias, visando a adoção das medidas de prevenção e controle (BRASIL, 2018).

A listagem das doenças de notificação compulsória nacional é estabelecida pelo Ministério da Saúde na Portaria de Consolidação n ${ }^{\circ} 4$ de 2017, e os dados coletados da notificação e investigação devem ser registrados no Sistema de Informação de Agravos 
de Notificação - SINAN, criado na década de 90, e que permite coletar, transmitir e consolidar dados, fornecendo informações para análise e interpretação dos dados (ROUQUAYROL, 1999).

A qualidade da informação em saúde depende da adequada coleta dos dados gerados no local onde ocorrem os eventos sanitários, como por exemplo, as Unidades de Saúde da Família (USF). É também nesse nível que os dados devem ser tratados e estruturados, para se constituírem em informação. A análise dos dados deve gerar informações de modo a desencadear ações de intervenção em tempo oportuno e com efetividade para o controle de doenças e agravos na população do território sob sua responsabilidade (BRASIL, 2018).

Assim, compreendendo a unidade de saúde como espaço prioritária e privilegiado para ações de vigilância epidemiológica, o presente trabalho tem como objetivo relatar a experiência de descrição das etapas do ciclo da vigilância em uma unidade de saúde no município de Salvador-BA, no contexto das vivências de campo do Programa de Treinamento em Epidemiologia Aplicada aos serviços do SUS.

\section{MÉTODO}

Trata-se de um estudo descritivo que relata a experiência do trabalho de campo de treinandos do Curso Epi-SUS, realizado em uma Unidade de Saúde da Família localizada no Distrito Sanitário da Boca do Rio, na cidade de Salvador - Bahia, no período de agosto a outubro de 2018. O município de Salvador possui extensão territorial de possui uma população estimada em torno de 2.938.092 habitantes. Integra a Região Metropolitana de Salvador (RMS), ao lado de mais 12 municípios, e está situada no Recôncavo Baiano, se constituindo no centro econômico, político e administrativo do estado da Bahia. Sua organização político-administrativa compreende 10 Regiões Administrativas denominadas Prefeituras Bairros e 12 Distritos Sanitários (DS), que são caracterizados como os territórios da saúde. Os Distritos Sanitários constituem-se em unidades de planejamento e gestão local dos serviços de saúde, seu território é definido por critérios populacionais, geográficos e epidemiológicos, e onde se localiza recursos de saúde públicos e privados (SALVADOR, 2018). 
Neste trabalho foi realizada entrevista semiestruturada com os membros da equipe de saúde da família após obtenção de autorização do Gerente/Coordenador do Distrito Sanitário. Para tanto foi utilizado formulário padronizado fornecido pelos tutores do curso. Por se tratar de um trabalho cujo objetivo era realizar treinamento de profissionais em especialização o presente não foi submetido ao comitê de ética conforme Resolução n ${ }^{\circ}$ 510/16 do Conselho Nacional de Saúde.

Desde 2000, a epidemiologia de campo no Brasil ganhou um novo capítulo com o Programa de Treinamento em Epidemiologia Aplicada aos Serviços do Sistema Único de Saúde (Epi-SUS). Já se passaram 15 anos de contribuições à saúde pública nacional e internacional, pois os resultados das ações do Epi-SUS foram amplamente divulgados e hoje fazem parte de livros, dissertações e teses que mudaram o paradigma da vigilância em saúde. Apesar das premiações nacionais e internacionais, é junto à população que a contribuição do Epi-SUS se faz mais presente. Sempre integrando a força-tarefa de resposta local de municípios, estados e países que enfrentam emergências de saúde pública, no Brasil e no mundo.

Os dados foram analisados utilizando a Matriz Strengths, Oportunities, Weaknesses, Threats (SWOT) e o Diagrama de Ishikawa. A Matriz SWOT ou FOFA em português, que é a sigla para Forças (Strengths), Oportunidades (Oportunities), Fraquezas (Weaknesses) e Ameaças (Threats), foi criada por Albert Humphrey, provavelmente na década de 60, na Universidade de Stanford (EUA). Através dessas variáveis, é possível analisar as forças e fraquezas de uma empresa e as oportunidades e ameaças do meio que a empresa atua. A matriz SWOT vem sendo usada para a gestão e o planejamento estratégico de uma organização por dar mais segurança para tomada de decisão, conhecer o cenário, antecipar-se a movimentos externos e indicar alternativas de ação (SOUZA, 2013).

O Diagrama de Ishikawa foi criado pelo professor Kaoru Ishikawa, da Universidade de Tóquio, também conhecido como Espinha-de-Peixe ou ferramenta de análise de causa e efeito é uma das ferramentas de qualidade mais utilizadas em saúde, auxilia determinar a origem de problemas, mostrando a relação do problema (causa) e os fatores que o influenciam (causas) (FIORIN, 2016). 


\section{RESULTADOS}

\section{MATRIZ SWOT}

A Matriz SWOT foi utilizada para detectar os pontos fortes e fracos inerentes a unidade de saúde e as ameaças e oportunidades que fogem a governabilidade da unidade para o cumprimento das ações do Ciclo da Vigilância.

\section{Tabela 1 - Resultados obtidos na Matriz SWOT}

\begin{tabular}{|c|c|}
\hline \multicolumn{2}{|c|}{ ANÁLISE SWOT } \\
\hline PONTOS FORTES & PONTOS FRÁGEIS \\
\hline $\begin{array}{l}\text { 1. Detecção passiva dos casos; } \\
\text { 2. Busca ativa dos casos pelos agentes } \\
\text { comunitários; } \\
\text { 3. Registro dos dados nos prontuários; } \\
\text { 4. Listagem das doenças de Notificação } \\
\text { compulsória disponível para consulta; } \\
\text { 5. Fichas do SINAN para notificação de } \\
\text { agravos e doenças; } \\
\text { 6. Disponibilização de testes rápido; } \\
\text { 7. Coleta de material biológico 1 vez por } \\
\text { semana na unidade; } \\
\text { 8. Disponibilidade de transporte para levar } \\
\text { amostras de baciloscopia ao laboratório } \\
\text { (motoboy); }\end{array}$ & $\begin{array}{l}\text { 1. Não realiza busca ativa nos prontuários; } \\
\text { 2. Inexistência do Guia de Vigilância } \\
\text { Epidemiológica; } \\
\text { 3. Ausência de notificação dos agravos } \\
\text { agudos; } \\
\text { 4. Déficit/Ausência de monitoramento das } \\
\text { doenças diarreicas; } \\
\text { 5. Ausência de análise de dados coletados; } \\
\text { 6. Distanciamento dos técnicos do Setor de } \\
\text { Vigilância Epidemiológica do Distrito } \\
\text { 7. Sanitário; } \\
\text { Fragilidade no feedback do Distrito. }\end{array}$ \\
\hline OPORTUNIDADES & AMEAÇAS \\
\hline $\begin{array}{l}\text { 1. Descentralização da Vigilância } \\
\text { Epidemiológica nos Distrito Sanitários; } \\
\text { 2. Laboratório Central Municipal para } \\
\text { análise das amostras; } \\
\text { 3. Ações de educação permanente nos } \\
\text { Distrito Sanitários. }\end{array}$ & $\begin{array}{l}\text { 1. Política de Recursos Humanos sem } \\
\text { perspectiva de contratações; } \\
\text { 2. Fragilidade no acompanhamento e } \\
\text { monitoramento dos técnicos da } \\
\text { Vigilância Epidemiológico do Distrito } \\
\text { Sanitário; } \\
\text { 3. Limitação da coleta de material biológico } \\
\text { na unidade. }\end{array}$ \\
\hline
\end{tabular}

Fonte: elaborado pelos autores, 2018.

\section{ESPINHA DE PEIXE}

O diagrama da Espinha de Peixe foi usado como metodologia para determinar as causas de um dos problemas apontados na Matriz SWOT. O entrave escolhido foi o déficit no monitoramento das Doenças Diarreicas Agudas por ser uma situação que envolve a sistematização de todas as etapas do ciclo da vigilância, deste a detecção até o encerramento dos casos. As possíveis causas do referido problema foram agrupadas em 
quatro categorias: Processos de trabalho, Profissional de Saúde, Gestão Distrital e Gestão local de Saúde.

Figura 1 - Causas para o déficit/ausência de monitoramento das doenças diarreicas agudas, Salvador, Bahia.

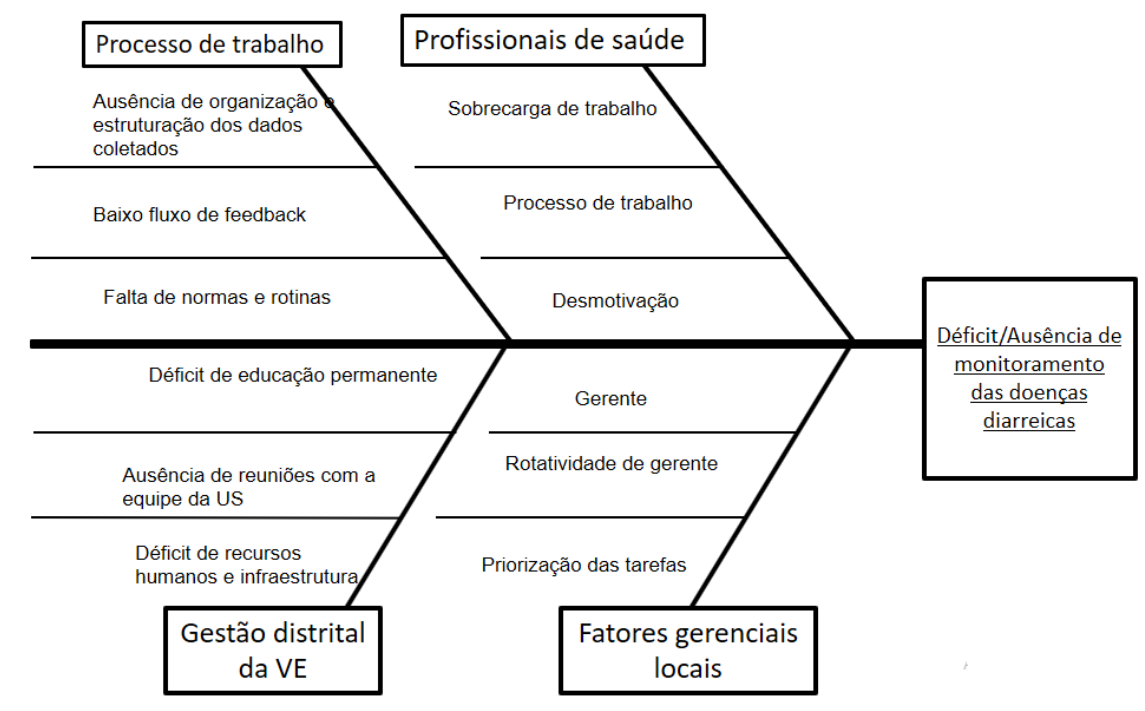

Fonte: elaborado pelos autores, 2018.

Depois de definir as possíveis causas, elas foram classificadas segundo seu grau de controle (Figura 3), usando a classificação: Totalmente dentro do seu controle para melhorar; parcialmente dentro do seu controle; não está no seu controle. 
Figura 3 - Classificação das causas para o déficit/ausência de monitoramento das doenças diarreicas agudas, segundo grau de controle, Salvador, Bahia, apontados no Diagrama Espinha de Peixe.

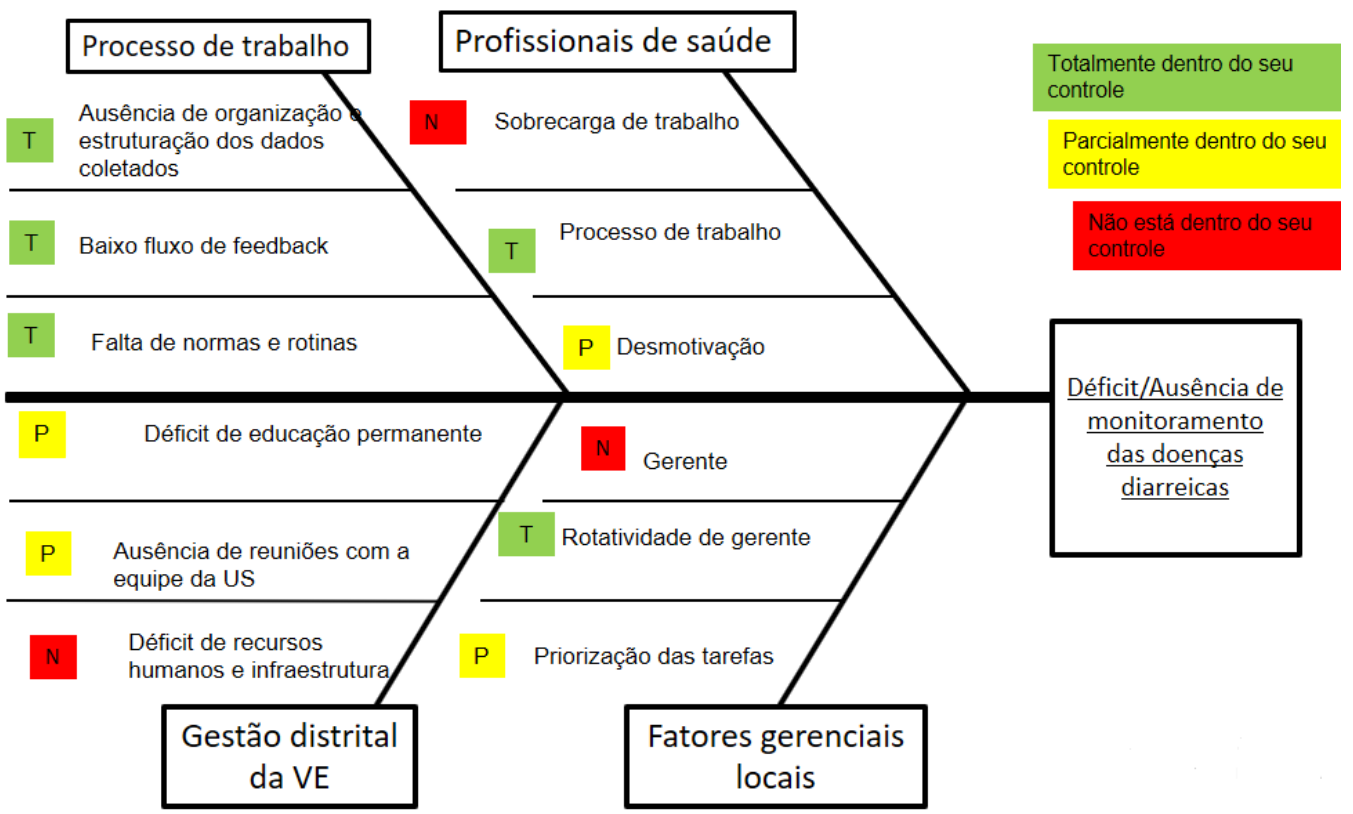

Fonte: elaborado pelos autores, 2018.

Com os achados dos resultados, visando a superação do problema em questão, foi realizada uma roda de conversa com a equipe da USF para devolutiva do trabalho e capacitações dos profissionais. O momento teve como objetivo promover reflexão sobre as fases do ciclo de vigilância em saúde, bem como apresentar recomendações para melhoria de qualidade das ações do mesmo.

\section{DISCUSSÃO}

A Atenção Básica no SUS reorganizou suas práticas de saúde através da estratégia da Saúde da Família, que tem como primícias adequar suas ações e serviços à realidade da população em cada unidade territorial (BRASIL, 2008). Assim, a avaliação da qualidade dos serviços ofertados é de fundamental importância para a constante melhoria dos mesmos e adaptação a realidade da população. Nesse sentido, é preciso considerar que a visão de profissionais e usuário sobre o serviço de saúde é carregada de aspectos 
subjetivos, incluindo uma carga histórica, cultural, política e ideológica, sendo a presença de observadores externos, como os pesquisadores, uma alternativa para uma melhor compreensão da realidade da unidade. (MARTINS, 2014).

Com relação a USF em estudo, observa-se a existência de diversos desafios (internos e externos) inerentes ao SUS em todo Brasil. Em especial, pode-se perceber a concentração de tais dificuldades em aspectos estruturais e com relação aos recursos humanos. Nesse aspecto cabe refletir sobre os desafios relacionados ao financiamento do SUS desde sua fundação e que perduram ainda hoje. Apesar dos avanços, como a aprovação da Emenda Constitucional (EC) nº 29/2000 que definiu valores mínimos a serem aplicados por cada ente federativo, houve retrocessos como a aprovação da $\mathrm{EC} \mathrm{n}^{\mathrm{o}}$ 95/2016 que definiu limites para aumento dos gastos federais nas diversas áreas, incluindo o setor saúde (CASTRO, 2018).

Em se tratando da Atenção Básica $(\mathrm{AB})$ a questão do financiamento também se mostra extremamente relevante, uma vez que ocupa papel central no ordenamento do cuidado e da Rede de Atenção à Saúde (RAS). Assim, o sucateamento por vezes encontrado em algumas USF - envolvendo recursos materiais, humanos e estruturais demonstra uma visão de $\mathrm{AB}$ como oportunidade de redução de custos do setor saúde (se comparado aos gastos com os níveis secundário e terciário) em detrimento de um modelo de atenção à saúde (CASTRO, 2018).

Os profissionais de saúde devem estar treinados e preparados para detecção de agravos e doenças, coleta e notificação de casos, análise e interpretação de dados e produção de informação em saúde, desempenhando importante papel nas ações de vigilância epidemiológica e contribuindo para o controle de doenças e agravos na população do território sob sua responsabilidade.

No entanto, quando se analisa as ações de vigilância epidemiológica desenvolvidas na Unidade de Saúde em questão, observa-se que muitas etapas do ciclo da vigilância não são realizadas porque não estão inseridas no cotidiano da prática dos profissionais de saúde.

Existem indícios que tal situação decorra da falta de contato com o tema durante a formação acadêmica e falta de treinamentos para os profissionais. Assim, o desconhecimento sobre leis e normas técnicas relacionadas ao processo de vigilância e notificação agrava o problema dentro da atenção básica. Além disso, diversos profissionais visualizam o processo de vigilância como ação exclusiva da equipe de 
vigilância epidemiológica do distrito sanitário e não como parte inerente e essencial para o cuidado. Aliada a essa falta de compromisso por parte de alguns profissionais, existe uma fragmentação no processo de vigilância entre as categorias profissionais, de modo que por vezes o processo acaba por não ser concluído de maneira efetiva (VICTOR et al., 2003).

A USF alvo do estudo realiza detecção passiva e ativa (através dos agentes comunitários) dos casos suspeitos, porém os dados coletados são registrados nos prontuários dos pacientes, dificultando à organização sistemática dos dados, necessária a consolidação dos dados para posterior análise e disseminação de informação sobre eventos de saúde.

As Fichas de Notificação são utilizadas apenas para agravos e doenças crônicas, como: casos de tuberculose, hanseníase e anemia falciforme. Os casos suspeitos de agravos ou doenças agudas são encaminhados para atendimento na unidade de emergência, onde são notificados. Essas são encaminhadas para o distrito sanitário, para o processamento eletrônico do SINAN, e o distrito por sua vez, não realiza o feedback dos resultados.

Na USF, nenhum outro instrumento de coleta de dados é utilizado a fim de organizar e categorizar dados de identificação, demográficos, laboratoriais, clínico, contatos, etc. de surtos ou outros eventos de saúde.

A falha apresentada no processo de notificação dos agravos e de concretização do ciclo da vigilância, com promoção inclusive de feedbacks pode estar associada a falta de assimilação das informações, bem como ao não entendimento da relevância do caso e da necessidade de notificação (LIMA, 2018).

\section{CONCLUSÕES}

Portanto, observa-se que a sistematização dos dados epidemiológicos - que deveriam ser interpretados e atualizados rotineiramente pela equipe de saúde da família - ainda é ação realizadas apenas por técnicos do Distrito Sanitário ou da Diretoria de Vigilância da Secretaria Municipal de Saúde de Salvador.

Sendo a unidade básica, o local privilegiado para desenvolver ações de vigilância, deve constituir-se em um espaço de articulação de conhecimento e técnicas vindos da 
epidemiologia, do planejamento e das ciências sociais, buscando uma prática de saúde que garanta a promoção da saúde, a prevenção, o tratamento e a reabilitação da população, das famílias, da comunidade.

Por fim, ressalta-se a importância da atividade de campo do Epi-SUS, enquanto atividade extensionista, para o desenvolvimento de uma reflexão crítica sobre a realidade por parte dos treinandos. Ademais, através de tais atividades espera-se também que esses tornem-se protagonistas em ações que visem a análise de problemas de saúde pública, propondo recomendações de melhorias que sejam efetivas e possíveis de colocar em prática na vigilância local. 


\section{REFERÊNCIAS}

BRASIL. Ministério da Saúde. Organização Pan-Americana da Saúde. Ministério da Saúde. EpiSUS - "Além das Fronteiras": Contribuindo para o Fortalecimento da Epidemiologia Aplicada aos Serviços do SUS. Brasília, DF: OPAS, Ministério da Saúde, 2015.

BRASIL. Ministério da Saúde. Secretaria de Atenção à Saúde. Departamento de Atenção Básica. Vigilância em Saúde: Dengue, Esquistossomose, Hanseníase, Malária, Tracoma e Tuberculose. Série A. Normas e Manuais Técnicos. Cadernos de Atenção Básica. n. 21. Brasília, 2008.

BRASIL. Ministério da Saúde. Secretaria de Vigilância em Saúde. Departamento de vigilância das Doenças Transmissíveis. EpiSUS-Fundamental: guia do tutor: oficina 1/Min Ministério da Saúde. Secretaria de Vigilância em Saúde. Departamento de vigilância das Doenças Transmissíveis . Brasília: Ministério da Saúde, 2018.

BRASIL. Resolução n. 588, de 12 de julho de 2018. Disponível em: https://www.jusbrasil.com.br/diarios/198910537/dou-secao-1-12-07-2018-pg-365

CASTRO, A. L. B.; et al. Financiamento da Atenção Primária a Saúde no Brasil. In: MENDONÇA, M. H. M.; et al. (org). Atenção Primária à Saúde no Brasil: conceitos práticas e pesquisa. 1. ed. Rio de Janeiro: Editora Fiocruz, 2018.

FIORIN, J. M. A.; et al. Uso do diagrama de Ishikawa associado ao planejamento estratégico: experiência na graduação em enfermagem. REVISTA UNINGÁ REVIEW, [s.l.], v. 26, n. 3, jun. 2016. Disponível em: http://revista.uninga.br/index.php/uningareviews/article/view/1809 - Acesso em: 24 mai. 2020.

LAZARINO, M. S. A.; SILVA, T. L.; DIAS, E. C. Apoio matricial como estratégia para o fortalecimento da saúde do trabalhador na atenção básica. Rev. bras. saúde ocup. v. 44, 23. ed., São Paulo: 2019. Disponível em: http://www.scielo.br/scielo.php?script=sci_arttext\&pid=S0303-

$76572019000100301 \& \operatorname{lng}=e n \& n r m=i s o$. Acesso em: 17 jul. 2020.

LIMA, R. K. S.; et al. Notificação compulsória de acidentes de trabalho: dificuldades e sugestões dos profissionais de saúde em Fortaleza, Ceará. Revista Brasileira de Medicina do Trabalho, [s.l.], v. 16, n. 2, p. 192-198, 2018.

MARTINS, L. F. V.; MENEGHIM, M. C.; MARTINS, L. C.; PEREIRA, A. C. Avaliação da qualidade nos serviços públicos de saúde com base na percepção dos usuários e dos profissionais. RFO UPF [online]. v.19, n.2, p. 151-158. 2014.

ROUQUAYROL, M. Z.; FILHO, N. A. Epidemiologia \& Saúde. 5. ed. Rio de Janeiro: MEDSI, 1999.

SALVADOR, Secretaria Municipal da Saúde do Salvador (SMS SSA). Diretoria Estratégica de Planejamento e Gestão (DEPG). Plano Municipal de Saúde do

Salvador 2018-2021. Aprovado pelo Conselho Municipal de Saúde em 21 nov. 2018. $231 \mathrm{p}$. 
SOUZA, L. P.; et al. Matriz swot como ferramenta de gestão para melhoria da assistência de enfermagem: estudo de caso em um hospital de ensino. Gestão e Saúde, v. 4, n. 1, p. 1633-1643, 2013.

VICTOR, J. F.; et al. Vigilância Epidemiologia em unidade básica de saúde da família. Rev Rene, Fortaleza, p. 46-52, dez. 2003.

WALDMAN, E. A. Vigilância em Saúde Pública. Colaboração de Tereza Etsuko da Costa Rosa. São Paulo: Faculdade de Saúde Pública da Universidade de São Paulo, v. 7. Série Saúde e Cidadania. 1998. 